\title{
Web Based Presentation of Semantically Tagged 3D Content for Public Sculptures and Monuments in the UK
}

\author{
Karina Rodriguez-Echavarria*, David Morris ${ }^{\dagger}$, David Arnold \\ Faculty of Management and Information Sciences, University of Brighton, UK
}

\begin{abstract}
The documentation and presentation of 3D digital content is a critical but non-trivial task for the Cultural Heritage sector. $\mathrm{Cu}$ rators are often faced with the task of cataloguing every piece of heritage and maintaining the resulting information in such a way which is suitable for scholarly research and public dissemination. Hence, the integration of $3 \mathrm{D}$ content poses additional challenges. This paper introduces research conducted to integrate semantically tagged 3D content to the catalogue acquired within the Public Monuments and Sculpture Association's National Recording Project (NRP) in the UK. This research involves the combination of graphical APIs and semantic technologies in order to integrate $3 \mathrm{D}$ content with semantic tags in a web browser. Although the initial results are still experimental; it is expected that they will support scholarly research and public dissemination by presenting a variety of integrated documentation on the project website: http: / / www.publicsculpturesofsussex.co.uk
\end{abstract}

CR Categories: H.3.5 [Online Information Services]: Web-based services- [H.3.7]: Digital Libraries- [I.3.8]: Applications-;

Keywords: Digital Library, Web based 3D, Semantic Technologies, Sculptures

\section{Introduction}

The Public Monuments and Sculpture Association's National Recording Project (NRP) for Sussex in the United Kingdom (UK) was established to catalogue every piece of public sculpture and every public monument in the area of Sussex and to maintain the resulting information in digital form for public access. The ongoing documentation stage involved a survey of the diverse range of monuments and sculptures in the cities, towns and villages of Sussex.

The survey includes, from all periods, conventional statues, monumental works, selected war memorials, architectural sculpture, art sculpture, memorial clocks, towers and fountains (see some examples in figure 1). These include those works that have 'disappeared', with diverse overall condition, under risk, or have some heritage listing. The detailed descriptive data (i.e. location, measurements, condition) is recorded as well as the objects' general history, including that of inauguration events/unveiling ceremonies. As a result, a variety of information is being documented into a

\footnotetext{
*e-mail: K.Rodriguez@brighton.ac.uk
}

†e-mail: D.Morris@brighton.ac.uk catalogue which is being made available from the project website: http://www.publicsculpturesofsussex.co.uk
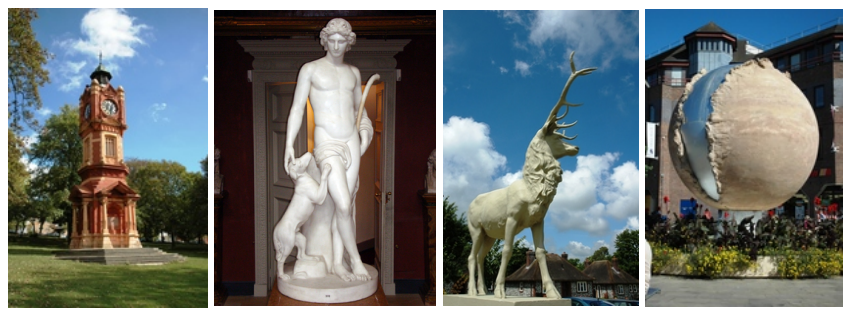

Figure 1: Examples of public monuments and sculptures in Sussex, UK. From left to right: Preston Park Clock Tower, Brighton; Pastoral Apollo, Petworth House, Petworth; The Diplock Stag, Polegate; Cosmic Cycle (The Rising Universe), Horsham

Within this context, the aim of this research is to incorporate the documentation of 3D digital representation of sculptures and monuments in the database. This aim involves the following novel objectives:

1. To integrate $3 \mathrm{D}$ digital assets with other types of documentation in order to enrich the catalogue.

2. To present the integrated content (3D data and metadata) in the website for scholarly research and public dissemination.

This paper will present the research conducted towards achieving these objectives using a combination of 3D graphics, semantic and web technologies. Section 2 will introduce the research background, while section 3 and 4 will describe the technological framework and a user-case of the development. Finally Section 5 and 6 will present further work and conclusions.

\section{Research background}

In order to address the objectives of the research, existing approaches for tagging 3D digital assets with other types of documentation and presenting this content through the web were researched. For this, it was necessary to take into account the needs of the Cultural Heritage sector. The requirements for semantically tagging $3 \mathrm{D}$ content were discussed with the curator of the NRP collection. They can be summarized as follows:

- To be able to structure the semantic meaning of elements of the sculpture in such a way that they could be related to parts of the 3D mesh by using semantic tags.

- To be able to easily and efficiently visualize 3D representations of sculptures and monuments in Sussex. This was regarded to be very valuable for scholarly research and public dissemination. The reason is that not all users of the collection have the same expertise for reading lengthy descriptions on the physical parts, history and iconographical meaning of each of the objects. Thus, the 3D content could be used to contextualise other information in the catalogue. 
The statue of Queen Victoria in the city of Brighton will be used to exemplify this set of requirements. For this statue, the database contains the following description:

"Octagonal base constructed of three graduated sections. Scrolls on the corners of the plinth and a rope border around the top plinth. She clutches a scroll (damaged) in her left hand and her robe in the right hand. She wears the Garter sash and her crown is worn over a mantilla. She is depicted in the act of opening Parliament."

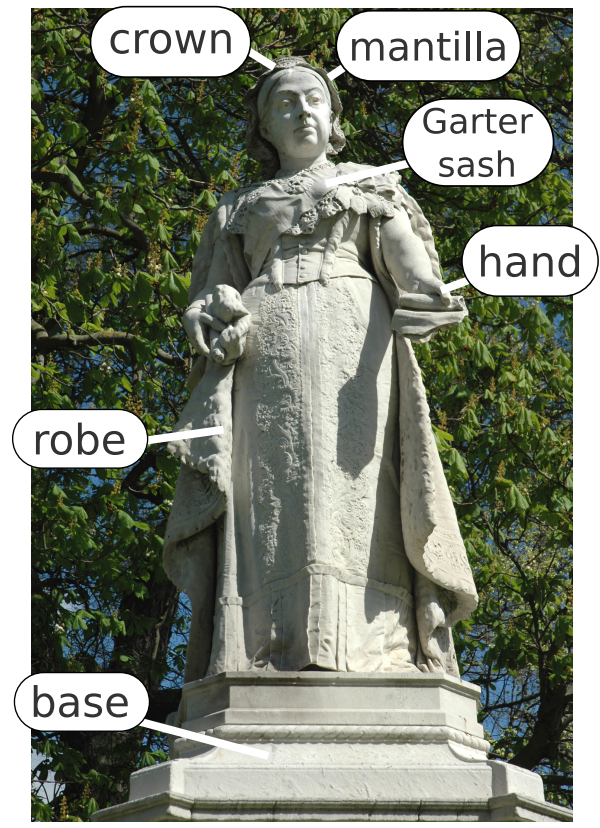

Figure 2: Elements of the sculpture used for describing the object

This description refers to different elements in the sculpture, such as crown, mantilla, Garter sash, hand, robe, base, as shown in figure 2. Each of these elements have a particular meaning as well as a significance to the overall sculpture. These additional information is known in the field as metadata. In order to retrieve these meanings, the information needs to be structured so it can be tagged to parts of the 3D mesh and visualised in a web browser. Furthermore, references to the metadata in other databases is possible. For example, a detailed description of the element "robe" might be found in sites such as wikipedia where additional meaning is explained.

There are several existing ontologies for structuring heritage information, such as Dublin Core and CIDOC-CRM. Additionally, initiatives such as the Text Encoding Initiative [Text Encoding Initiative 2009] provides standard for the representation of texts including those in the cultural heritage sector. In particular, CIDOC-CRM is a formal ontology which facilitates the integration and interchange of heterogeneous cultural heritage information [Doerr and Iorizzo 2008]. One of the main characteristic of this ontology is that it analyses the past dividing it into discrete events involving persistent items, both material and immaterial. It is the material items which are of interest for this research, as they provide the link between the 3D digital representation and its related documentation.

Although research related to the presentation of $3 \mathrm{D}$ content in the web is abundant, its integration with current semantic technologies is still a challenging area. Previous research in using ontologies includes adding semantic annotations to areas of the 3D model [Bilasco et al. 2006], for instance to describe what the geometry rep- resents. [Pittarello and De Faveri 2006] proposed an approach for associating semantic information to 3D worlds using the X3D language. This semantic information involved mainly naming what an object in the 3D scene represents without further links to additional information.

Commercial tools, such as Adobe Acrobat 3D [Adobe Systems Incorporated 2009] have also developed mechanisms for embedding $3 \mathrm{D}$ content into PDF documents. This approach enables the annotation of the 3D model with textual tags. However, its disadvantage is that it requires a commercial reader from Adobe to access the functionality instead of integrating the functionality within the web browser along with other types of content.

Recent work in the area includes [Havemann et al. 2008], which developed an exemplary authoring tool for generating a Collada lite based scene description which includes the 3D model along with semantic annotations. The annotations are then linked to markedup areas in the model. As a result, it is possible to interact with the 3D model and browse the semantic links contained in the model. However, the annotations are only textual tags and do not provide a fail-safe entry point to a metadata repository. In addition, the interactions with the 3D model are not integrated within a web browser instead a presentation tool with an integrated web browser is used. Our approach builds on these ideas following similar mechanisms to tag the semantic information. Nevertheless, the main innovation is that the research uses web technologies to integrate the 3D model with its CIDOC-CRM based tags to additional metadata in a web browser. As a result, every tag is an entry point to additional metadata in the CIDOC-CRM repository, which can be searched within the same webpage of the project. This will be explained in detail in the following sections.

\section{System architecture for the dissemination of Public Sculptures and Monuments in the UK}

A system architecture based on standard web technologies was developed in order to acquire, document and enable public access to the NRP Sussex catalogue. Figure 3 illustrates this architecture consisting of three layers:

1. Content repositories for multi-modal information of the collection (text, images, 3D content).

2. Web Server based technologies to query and present the content.

3. Browser client for the users, including the curator, scholar researchers and the public.

Using this architecture, the users are only required to access the NRP website to add, edit, query and interact with the different types of content. In order to use the 3D functionality, the user needs to install a plug-in. The rest of the interactions with the 3D model are provided by Javascript, AJAX and PHP technologies. They dynamically generate pages with the metadata related to the $3 \mathrm{D}$ content. The metadata might be within the repository of the project or other repositories.

The following subsections will describe: i) the approach used for integrating 3D content with semantic tags as well as ii) presenting this content using web technologies within this system architecture.

\subsection{Semantic tagging of 3D content}

The content initially acquired for the catalogue involved mainly text and photographs of the sculptures and monuments. This content 


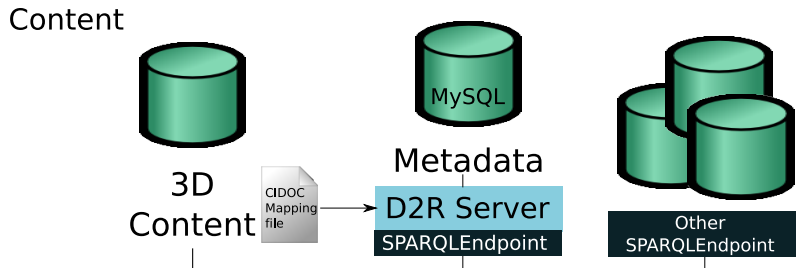

Web server

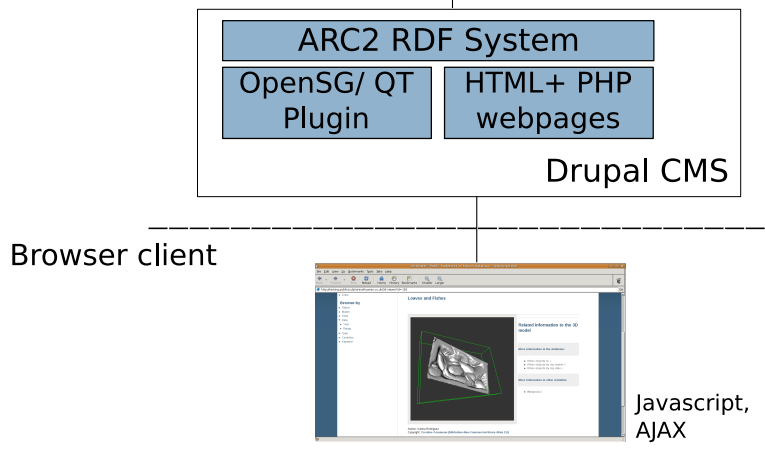

Figure 3: System architecture for presenting semantically tagged 3 content

was stored in a MySQL database and provided the basis for generating the metadata for the $3 \mathrm{D}$ content. For this, the CIDOC-CRM [International Council of Museums 2009] ontology was selected to provide a mapping of this documentation. This approach was followed as the current structure of the database is proprietary and does not ensure interoperability with other repositories. This is particularly an issue when considering other repositories of sculptures and monuments in the UK. Currently, each database has a proprietary structure which suits the local creators, but makes it noninteroperable with other repositories. Hence, finding a common language will provide a mechanism to ensure the interoperability of all databases in the future.

The D2R Server [Bizer and Cyganiak 2009] tool was used for publishing the content of the MySQL database in RDF (Resource Description Framework) for the Semantic Web. The D2R server provides the mapping from MySQL to CIDOC-CRM on the fly. The advantage of this approach is that the curator only needs to maintain one database for the whole framework. However, the disadvantage is that the mapping incurs a performance cost; although this could potentially be batch processed and cached.

A mapping file was developed to map the database content into CIDOC-CRM concepts, and to allow the RDF data to be browsed and searched within this context. As such, the mapping file provided a translation for each of the relevant concepts in the database to a CIDOC-CRM complaint concept. This is shown in a small section of the mapping file in Notation 3 language below:

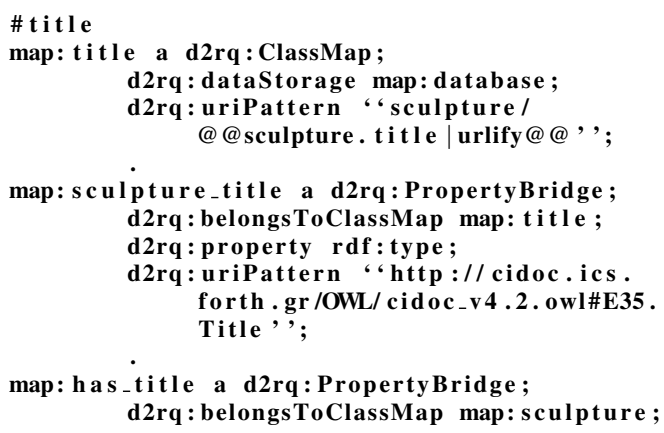

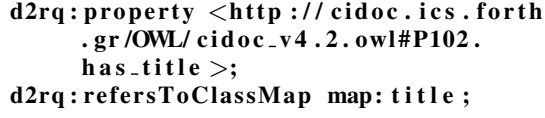

The code below specifies that the attribute "title" of the "sculpture" table in the database (sculpture.title) is equivalent to the entity E35 called "Title" in the CIDOC-CRM ontology. In addition, the property P102 called "has title" is used to specify the relationship between a sculpture and its title. Mapping this type of concepts (i.e. title, address, unveiling date) requires mainly looking for equivalent concepts in both the relational structure and the CIDOC-CRM ontology. Even more challenging is creating a mapping for concepts contained in text fields in the relational database, such as the description of the sculpture. This is because each description might contain different concepts; as described in the example of Queen Victoria in section 2. Future applications in semantic processing might help to automate this process; but for the moment the mapping has to be done manually and requires a close collaboration between the curator and developers.

Furthermore, an open source annotation tool called Tagg3D was developed to attach metadata to 3D content. This tool was developed using QT4 and the scenegraph API OpenSG2 [OpenSG 2009], which is a portable scenegraph system to create realtime graphics programs. Tagg $3 D$ enables users to interactively load a $3 \mathrm{D}$ scene, connect to a metadata repository, and attach tags to parts of the scene. This idea follows the approach proposed by [Havemann et al. 2008]. The innovation involves using a URIs (Uniform Resource Identifiers) instead of only text to provide an entry point to the CIDOC-CRM metadata repository.

As a result of the annotation process, Tagg $3 D$ creates a file containing the 3D mesh and associated URIs. The latter are later used within SPARQL queries to request more information. Hence, looking at the 3D content from a scenegraph perspective, each node in the scenegraph is linked to a tag containing a URI. In order to tag different parts of the geometry new nodes with their associated tags are created under the node which holds the overall geometry. As an example, the mapping shown before produces URIs of the form: http://donbot.itri.brighton.ac.uk:2020/ resource/sculpture/Loaves_and_Fishes. This URI is then used to link the 3D mesh of this sculpture to its metadata, as show in this COLLADA lite file:

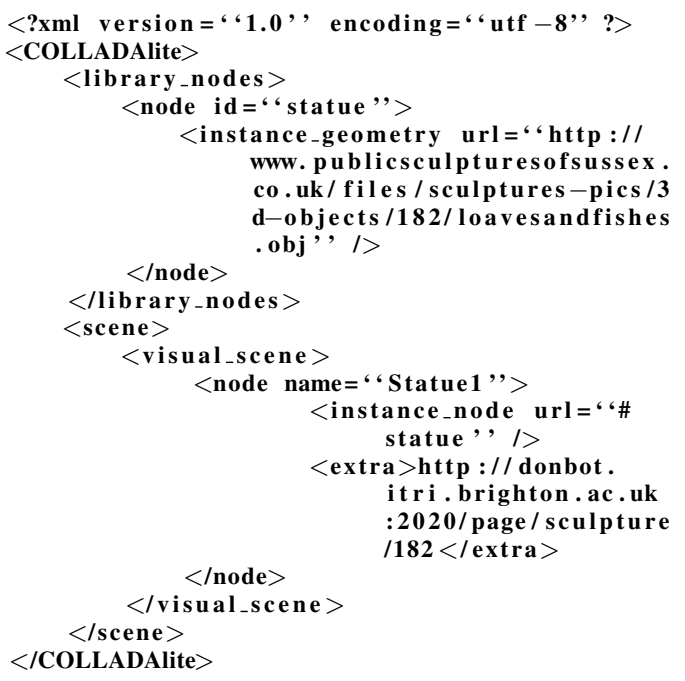

The metadata in the repositories is always queried using the SPARQL interface of the D2R server. This interface enables 
any application/website to search and query the database using the SPARQL query language over the SPARQL protocol. Within Tagg $3 D$ and the NRP website, the metadata is retrieved by making SPARQL queries using the URIs. In addition, queries could be done to other repositories with a SPARQL endpoint, such as dbpedia, to access additional information.

\subsection{Server and client side web technologies}

Initially, a functional site (www.publicsculpturesofsussex.co.uk) was developed for the NRP Sussex catalogue, which contained only access to the documentation including text and images. For this, the content management system Drupal was used. In this site, users are able to browser and access information on the sculptures and monuments in the catalogue. The pages are dynamically built using PHP based upon the records held within the project database. In addition, further functionality enables the curator of the collection to add and edit records for the various objects in the database. The advantage of using Drupal rather than a custom site is that the boilerplate development is already done such as user management, theming and layout.

Furthermore, it was required to enhance the website for any user to be able to interact with $3 \mathrm{D}$ content whilst visualising metadata associated with the content. Hence, experimental work is being conducted in order to enrich the website to provide these functionalities. These are currently being tested in a separate website for the project: http://testing. publicsculpturesofsussex.co.uk.

\subsubsection{D viewer plug-in implementation}

A 3D plug-in was developed using the scenegraph API OpenSG 2.0 [OpenSG 2009] and the browser plug-in implementation for QT4 [Nokia Corporation 2009]. This plug-in was built as the basis for rendering the $3 \mathrm{D}$ models in the webpage along with a user interface to present semantic information. This QT4 browser plug-in can be used in any web browser supporting the "npruntime" API, such as Netscape, Mozilla FireFox, Opera. The npruntime is an extension to the original NPAPI to supports plug-ins in browsers. This extension to the API enable the plug-in to be scriptable and access the scripted objects in the browser. Thus, it is a much robust and more flexible API [Mozilla 2009]. Current versions of Microsoft Internet Explorer do not support this API; but the Qt solution includes an ActiveQt framework to compile a single plug-in library that works with most web browsers.

The advantages of using an OpenSG based web plug-in over simply an OpenGL or composite window is that it provides access to the multiple functionalities already embedded in the scenegraph API. For instance, the loaders for different file formats, scene managers and rendering optimizations for the scene. Hence, the plug-in is not bound to current implementations of 3D for the web. As such, having one plug-in supporting different file formats is similar to having one media player plug-in supporting multiple video formats. Nevertheless, disadvantages include packaging the opensg libraries so the plug-in can be easily distributed and installed.

Currently, the 3D plug-in implements an OpenSG 2.0 passive window inherited from QGLWidget. The main functionalities of this window are:

- To display a 3D mesh, allowing user real-time interaction (rotate, move, zoom)

- To output tags with metadata associated to any part of the geometry in the form of a URI. The mechanism for achieving this were explained in the previous sub-section.
The plug-in includes two properties which along with the plug-in definition are defined as follows:

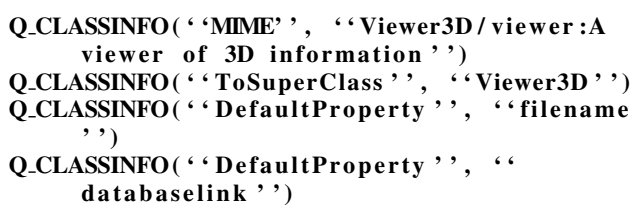

The use of this plug-in in the web page will be described in the following subsection.

\subsubsection{Website interface implementation}

Within the website, the page is structured as follows:

- 3D viewer: to display the 3D content

- Associated information area: to specify and interact with additional metadata

The page is dynamically built using PHP in order to create this interface. For this, the plug-in is called using the name of the 3D file associated to a certain object as follows:

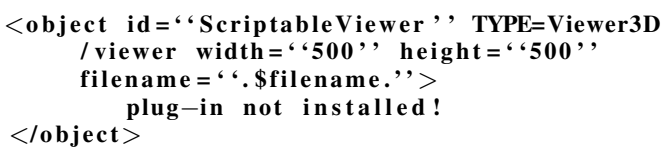

The 3D mesh is then loaded into the 3D plug-in in the viewer area. The file can have multiple links to metadata using the URIs described in the previous sections. Each of these URIs allows associated semantic information to be retrieved. As a result, when a section of the geometry is clicked on, its tag is stored in the variable called 'databaselink' within the $3 \mathrm{D}$ viewer plug-in. This variable is then accessed by the html page, in this case via a Javascript method which is called whenever the mouse is moved over the $3 \mathrm{D}$ plug-in as follows:

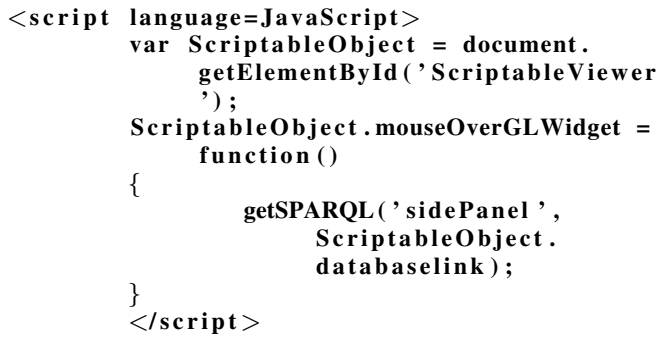

As shown in the code below, once the URI is retrieved by the html page it is used within a SPARQL query to populate the information area with the associated semantic information. The "Associated Information area" is then updated via an AJAX function allowing for content to be updated seamlessly as the user explores the 3D model. This enables the html page to update the associated metadata without reloading the $3 \mathrm{D}$ viewer. This works through the use of a wrapper functions written in PHP so that multiple sites can be queried via SPARQL, rather than directly in Javascript which would be blocked by the browser due to cross-site scripting.

The PHP page uses the Arc2 [Arc 2009] library for SPARQL queries which are built and executed based on the variables passed in. Once the results of the query has been returned, a basic html page with the information and formatting is generated and loaded into the "Associated Information area". The html is then read by the AJAX function for inclusion within the original webpage. 
The following section will describe how this technology was deployed using as a case study the "Loaves and Fishes" sculpture in Brighton, UK and its associated metadata.

\section{Case study: documentation process}

Within the NRP, monuments and sculptures were documented across Sussex based on a close inspection to the objects. In addition to this, few objects were selected to trial the acquisition, documentation and dissemination of 3D content in the catalogue. Among this, the "Loaves and Fishes" sculpture in the city of Brighton was selected. This is a large sculpture set into an open rectangle in the brick wall as shown in figure 4 . The sculpture depicts a hand holding a loaf of bread with intertwining fish above and below. The sculpture refers to the biblical story of Christ feeding the 5000 with five loaves and two fish.

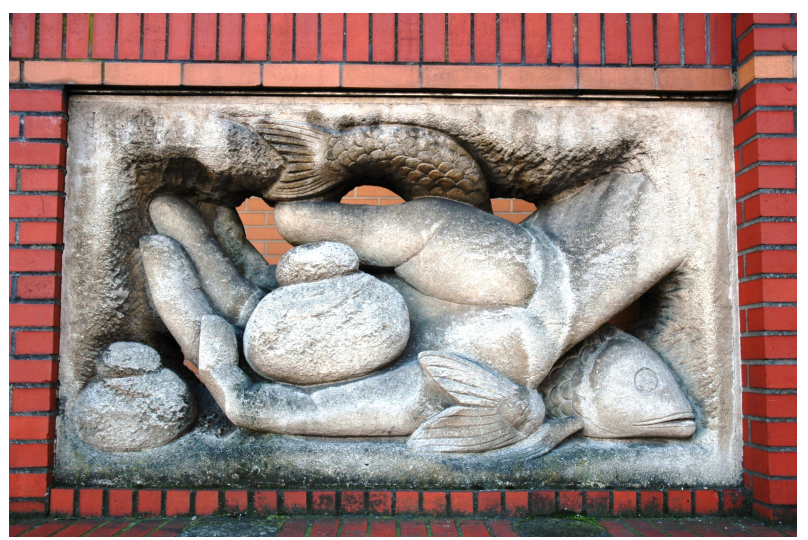

Figure 4: "Loaves and Fishes" public sculpture in Brighton, UK

\subsection{Acquisition and storage}

The textual information of the sculpture was acquired by visual inspection by the curator of the collection. This information was complemented by archived photographs and others taken by the curator on site. Further work was done to acquire the 3D information of the sculpture. The process for acquiring this information is still at an experimental stage. Hence, further work is being conducted to find an efficient and effective solution which could be deployed on a large scale basis for all objects in the database.

Currently, two techniques for acquiring the $3 \mathrm{D}$ information of several objects has been used:

- Expert acquisition using laser scanning: This process requires a laser scanner and expertise in handling the hardware and processing the $3 \mathrm{D}$ information.

- Non-expert acquisition using photogrammetry techniques: This process requires a digital camera and low to medium expertise in taking the pictures and processing the $3 \mathrm{D}$ information.

In the case of the Loaves and Fishes sculpture the non-expert acquisition technique was selected. This is because the sculpture is on a public building alongside a busy street. Thus, there is a large amount of daily passers-by which might obstructs the installation of the laser scanner and a more lengthy acquisition process. Therefore, a digital camera, the Arc3D service ([Vergauwen and Gool 2006], [K.U.Leuven ESAT-PSI 2009]) and MeshLab [Visual Computing Lab of ISTI-CNR 2009] were used.
The process involves mainly a non-expert user taking a series of digital photographs of the sculpture. These were then uploaded to a server where a 3D reconstruction of the scene was constructed. The MeshLab software was then used to clean and produce a simpler version of the $3 \mathrm{D}$ mesh of the sculpture suitable to distribute via the website. In this case, the resulting mesh is shown in figure 5. A poisson surface reconstruction [Kazhdan et al. 2006] approach has been used to build a reconstruction of the mesh resulting from Arc3D. The simplified version contains 63,090 vertices and was saved as an .obj file.

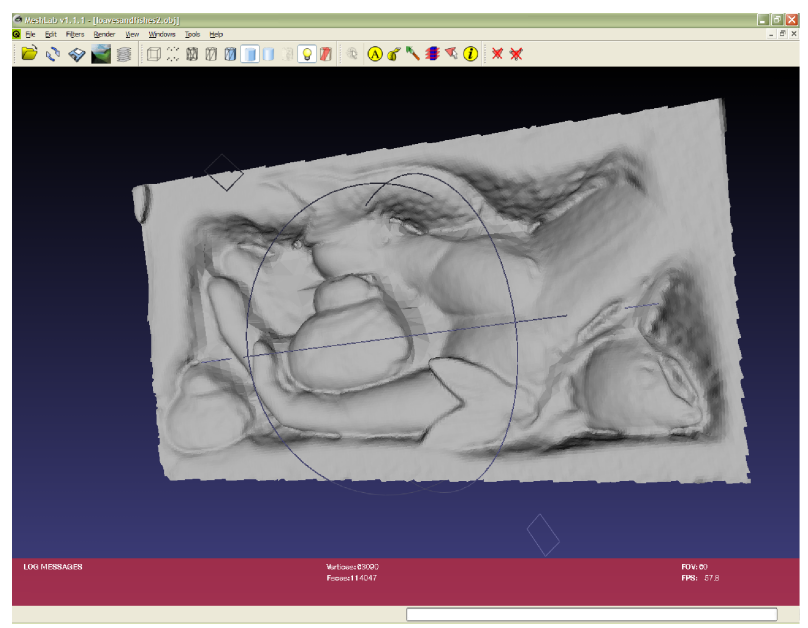

Figure 5: MeshLab software processing the Arc3D reconstruction of the sculpture

Using this process to create 3D content for the sculptures and monuments is more effective for a large scale digitisation exercise. Nevertheless, the experimental exercises have identified the need for a higher degree of expertise than initially expected. This is specially the case for taking good photographs and learning to use the postprocessing software. The experiments also identified that the photogrammetry technique works better for relieves, as exemplified by this sculpture, than for other types of sculptures. In particular, statues that contain a higher level of self occlusion and a higher level of detail is required to be captured.

\subsection{Tagging metadata to the 3D content}

Once the 3D mesh of the Loaves and Fishes was produced, the next step was to tag the mesh with links to the metadata available from the CIDOC-CRM compliant repository. As a general rule, it was decided to attach tags only for Persistent items within the CIDOCCRM concepts. This is because a 3D mesh can represent things such as Information Carriers (E84), and other things derived from Persistent Items (E77); but not Places, Dimension, Time-span or Events. All of the latter are always refered to by Persistent Items.

The selected tags for this sculpture were related to its iconographical description, which reads as follows:

"Refers to Christ feeding the 5000 with but five loaves and two fish. (Matthew 14:1321, Mark 6:31-44, Luke 9:10-17 and John 6:5-15)"

From this description we identified one tag for the mesh. This tag links the overall sculpture to an Information Carrier (E84) CIDOCCRM entity. As shown in figure 6, this entity contains a couple of properties. Within CIDOC-CRM ontology, a property serves to define a relationship of a specific kind between two classes. Hence, 
the property $\mathrm{P} 102$ called has title relates the Information Carrier 182 to its Title (E35) of Loaves and Fishes. In addition, the property P62 called depicts (is depicted by) specifies the relationship with the event (E5) of Christ feeding the 5000 with but five loaves and two fish. This event is documented in (P70) four different documents (E31). All this contextual information is linked by just attaching the following tag to the 3D file: http://donbot.itri. brighton.ac.uk:2020/page/sculpture/182

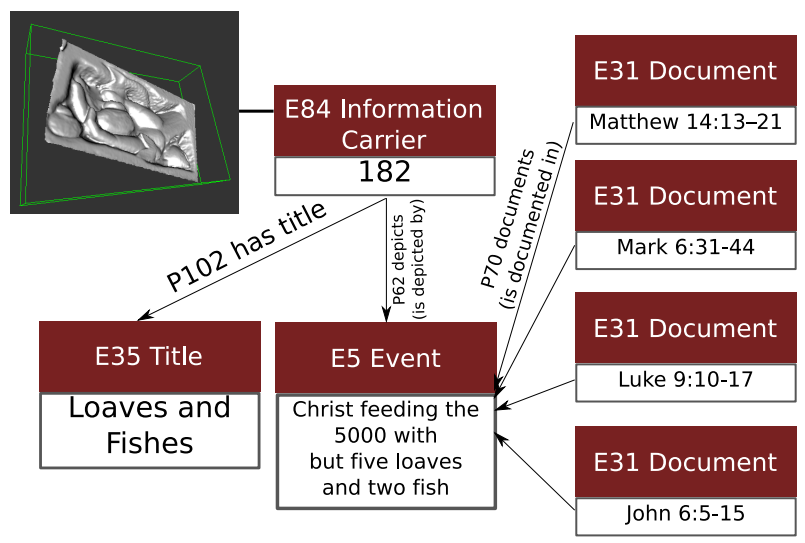

Figure 6: CIDOC-CRM semantic metadata linked to the $3 D$ content

The attachment of the tag to the mesh was done using the Tagg $3 D$ tool (see figure 7). The tool allows the user to specify metadata repositories, which are queried to extract tags in the form of URIs for the geometries within the scene. After the file is loaded into the scene and the geometry is selected, the tool allows the user to open a metadata dialogue window. A list of tags is then presented to the user, who selects the one that provides the best link to the metadata. For this, the tool makes a SPARQL query to the repository which has been previously specified. Currently, the tool creates basic queries, such as getting all persistent items from the metadata repository. However, it is possible to create more complicated queries.

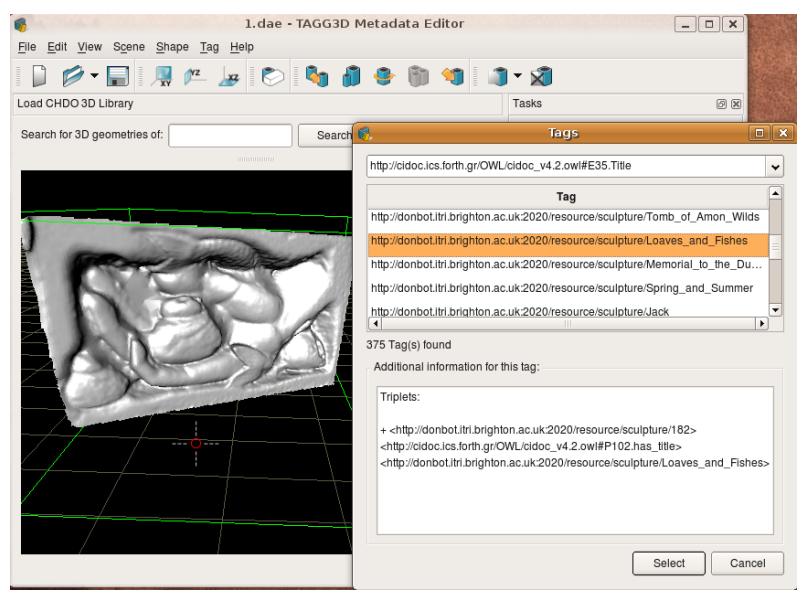

Figure 7: Tagg3d tool to annotate $3 D$ content with metadata

As a result, the tool produces a COLLADA lite file which is stored along with the images of the sculpture in the web server. The resulting file is shown in section 3.1 and contains the $3 \mathrm{D}$ file and the metadata tag.

\subsection{Web presentation of semantically tagged 3D con- tent}

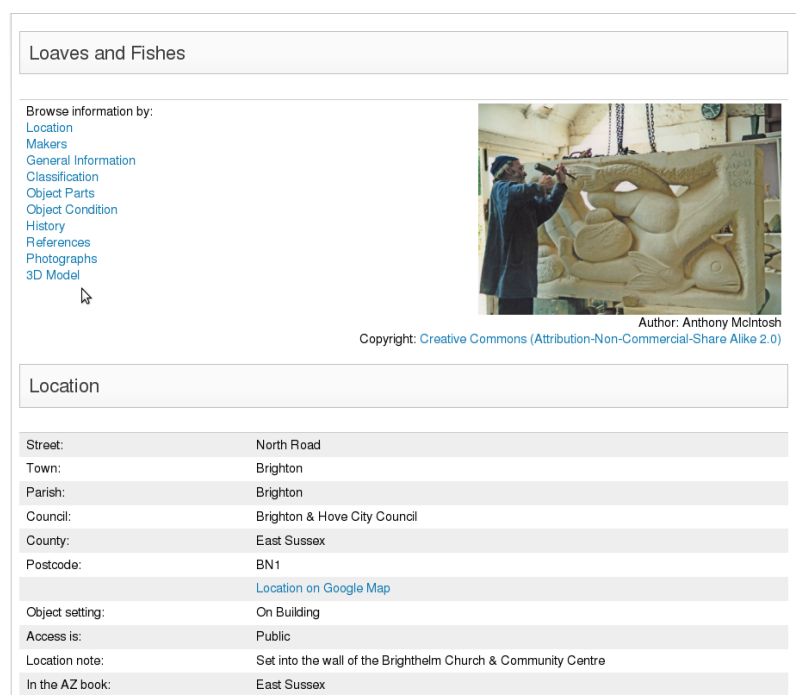

Figure 8: Web-page interface for monuments and sculptures in the NRP Sussex catalogue

The experimental work for the 3D content is currently being conducted within the testing website for the NRP Sussex catalogue. This interface is similar to the current working site. The only additional functionality is the access to $3 \mathrm{D}$ content along with other types of information. The site offers the functionality to browse the catalogue by object title, maker, town, date of unveiling or installation, type, condition or free keyword search. Once the user has found the object of his/her interest, a page with all detail information is presented. This page for the Loaves and Fish sculpture is shown in figure 8 . The information presented contains key information, such as address and location in google maps, information on the makers, classification, object parts, condition, history, references, photographs and the link to the 3D model.

The 3D model link gives access to the webpage with the plug-in, which can be downloaded if it has not been previously installed in the browser. More efficient mechanisms to perform this installation are currently being built, as currently it is not a straight-forward process. Once installed, the user can see the 3D model in the OpenSG based $3 \mathrm{D}$ viewer. Basic functionalities of the plug-in allow the user to move, rotate as well as zoom in and out in order to manipulate the geometry.

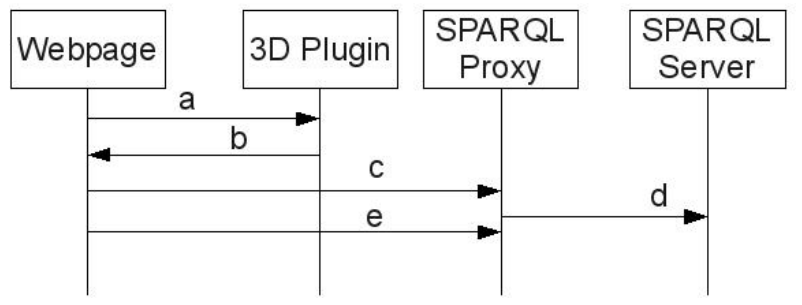

Figure 9: Sequence diagram showing the interactions which takes place when a user clicks on a tagged area of the Loaves and Fishes $3 D$ mesh.

Figure 9 shows the interaction between the components of the system. Firstly, a javascript call (a) is made to the 3D plug-in to load 
the Loaves and Fishes .obj file. Once a user clicks on the 3D mesh, the 3D plug-in updates the shared variable with the URI tag associated to the metadata which the user is interested in retrieving (b). In this case the URI http: / / donbot.itri.brighton. ac.uk:2020/page/sculpture/182 is retrieved. The URI is then used to request additional information from the SPARQL proxy $(\mathbf{c})$, which in turn requests the data from the CIDOC-CRM mapping (d). Once this has completed and generated a html response, it is loaded onto the webpage (e).

Once the metadata is retrieved, this is displayed in the browser in a way which allows users to easily navigate and explore as shown in figure 10. For this, a table with the properties and entities is displayed along with links which allow the user to explore semantic content on the metadata repository.

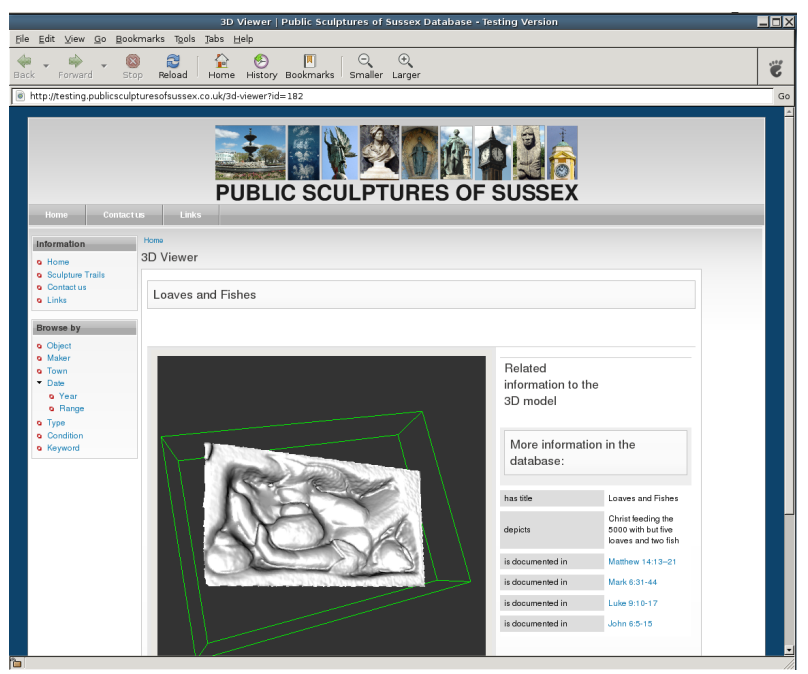

Figure 10: Web-page showing the $3 D$ model and semantic information for the Loaves and Fishes

The use of semantic information linked to the $3 \mathrm{D}$ content provides for a different browsing behaviour to navigating the site through more ridged options which were previously implemented. For example, in the current working website it is only possible to view the information of the sculpture without overlooking at the contextual visual information in the object. With this approach, it is now possible to describe visually the object and link together metadata through the semantic network.

\section{Further work}

Further work is expected to progress in three areas. Firstly the data capture of sculptures will progress providing for a more comprehensive test bed of 3D models. For this, it is necessary to create a simpler process which could be deployed by a small group of experts supporting a larger group of volunteers who live within the Sussex area.

Secondly, further work will be conducted to simplify the installation of the plug-in into multiple browsers. This will facilitate the use of the 3D model within the working website and will further contribute to the take-on of this approach to display 3D content on the web.

Thirdly, the semantic information associated with each object will be enhanced. This will be accomplished by enriching the mapping between the D2R server and MySQL. Also by enabling an userfriendly navigation within the metadata repository based on seman- tic tags, such that users can find additional information which they are interested in

\section{Conclusion}

This paper has presented ongoing research to integrate 3D content with semantic information for public sculptures in Sussex, UK which have been documented as part of the National Recording Project. The collected metadata for the various sculptures was used for the creation of the on-line database which is accessible via the web-site: http://www. publicsculpturesofsussex.co.uk

The mechanism for tagging was developed via an application called Tagg $3 D$ which links parts of a 3D geometry to CIDOC-CRM URIs in order to store entry points to a metadata repository. Although this metadata is currently structured using the standard CIDOC-CRM; it is possible to use other ontologies as long as the mechanism provided for querying uses a SPARQL endpoint. The current architecture uses the D2R server to store the CIDOC-CRM mapping to the metadata by extracting the data from MySQL via a mapping file.

Within the testing web-site, 3D models can be viewed via the 3D plug-in which was developed using Qt4 and OpenSG 2.0. 3D content is displayed either as a single model or a more complicated model comprised of multiple sections connected via a scene-graph.

All of this combines into a simple to use web-site which after the installation of a plug-in, allows a user to interact with a 3D model of a sculpture whilst exploring both textual and semantic information relating to it.

\section{Acknowledgements}

This work has been conducted as part of the 3D-COFORM Integrating Project (FP7-ICT-231809) and the National Recording Project for Sussex funded by the Heritage Lottery in collaboration with the Public Monuments and Sculpture Association. Our thanks to Anthony McIntosh, Research Officer for the National Recording Project for Sussex. Thanks are also due to the Computer Graphics and Knowledge Visualisation Group at the Graz University of Technology for their support on the implementation of COLLADA lite for storing metadata for 3D content. The 3D models presented in this paper were created by the ARC 3D webservice, developed by the VISICS research group of the K.U.Leuven in Belgium.

\section{References}

Adobe Systems Incorporated, 2009. Acrobat 3d, April. http: //www. adobe.com/devnet/acrobat 3d/.

ARC, 2009. Arc home page, April. http://arc. semsol. org/.

Bilasco, I. M., Gensel, J., Villanova-Oliver, M., AND MARTIN, H. 2006. An mpeg-7 framework enhancing the reuse of $3 \mathrm{~d}$ models. In Web3D '06: Proceedings of the eleventh international conference on $3 D$ web technology, ACM, New York, NY, USA, 65-74.

BIZER, C., AND CYGANIAK, R., 2009. D2r server, April. http://www4.wiwiss.fu-berlin.de/bizer/ d2r-server/.

Doerr, M., AND Iorizzo, D. 2008. The dream of a global knowledge network-a new approach. J. Comput. Cult. Herit. 1, $1,1-23$. 
HaVemann, S., Settgast, V., Berndt, R., YVind Eide, And Fellner, D. W. 2008. The Arrigo Showcase Reloaded towards a sustainable link between $3 \mathrm{D}$ and semantics. Eurographics Association, Braga, Portugal, M. Ashley, S. Hermon, A. Proena, and K. Rodriguez-Echavarria, Eds., 125-132.

InTERnATIONAL COUNCIL OF MUSEUMS, 2009. Cidoc crm home page, April. http://cidoc.ics.forth.gr/.

Kazhdan, M., Bolitho, M., And Hoppe, H. 2006. Poisson surface reconstruction. In SGP '06: Proceedings of the fourth Eurographics symposium on Geometry processing, Eurographics Association, Aire-la-Ville, Switzerland, Switzerland, 61-70.

K.U.LEUVEN ESAT-PSI, 2009. Arc3d home page, April. http: $/ /$ www $\operatorname{arc} 3 \mathrm{~d}$.be/.

MozILla, 2009. Scripting plugins, April. https: //developer.mozilla.org/En/Gecko_Plugin_ API_Reference: Scripting_plugins.

NoKia CORPORATION, 2009. Qt browser plugin, April. http: //www. qt software.com/products/appdev/ add-on-products/catalog/4/Utilities/ qtbrowserplugin.

OPENSG, 2009. http://opensg.vrsource.org/trac.

Pittarello, F., And De Faveri, A. 2006. Semantic description of $3 \mathrm{~d}$ environments: a proposal based on web standards. In Web3D '06: Proceedings of the eleventh international conference on 3D web technology, ACM, New York, NY, USA, 85-95.

Text Encoding Initiative, 2009. Webpage, April. http: //www.tei-c.org/.

VERGAUWEn, M., AND Gool, L. V. 2006. Web-based 3d reconstruction service. Mach. Vision Appl. 17, 6, 411-426.

Visual COMPUTING LAB OF ISTI-CNR, 2009. Meshlab home page, April. http://meshlab. sourceforge.net/. 